\title{
Geleitwort
}

\section{Tu felix Austria?}

\section{Geleitwort des Vorstands der Deutschen Gesellschaft für Psychologie zum Themenheft „Psychologie in Österreich“}

\author{
Birgit Spinath ${ }^{1}$, Christian Fiebach ${ }^{2}$, Annette Schröder ${ }^{3}$, Gordon Feld ${ }^{4}$, Ulrich Ansorge ${ }^{5}$, \\ Markus Bühner ${ }^{6}$ und Jens Bölte \\ ${ }^{1}$ Universität Heidelberg \\ ${ }^{2}$ Goethe-Universität Frankfurt am Main \\ ${ }^{3}$ Universität Koblenz-Landau \\ ${ }^{4}$ Zentralinstitut für Seelische Gesundheit Mannheim \\ ${ }^{5}$ Universität Wien \\ ${ }^{6}$ Ludwig-Maximilians-Universität München \\ ${ }^{7}$ Westfälische-Wilhelms Universität Münster
}

Die enge Zusammenarbeit zwischen der Psychologie in Österreich und Deutschland hat eine sehr lange Tradition. Gerade in diesem Jahr hätte diese Tradition durch die geplante zeitgleiche Ausrichtung der Kongresse von DGPs und ÖGP in Wien in besonderem Maße mit Leben gefüllt werden können und uns die Möglichkeit gegeben, den Austausch zu verstärken. Die Corona-Pandemie hat uns leider einen Strich durch diese Rechnung gemacht. Aus diesem Grund freuen wir als Vorstand der DGPs uns in besonderem Maße darüber, durch das hier vorliegende Sonderheft „Psychologie in Österreich“ einen aktuellen Einblick in die akademische und berufspolitische Situation in unserem Nachbarland zu erhalten. Die länderübergreifenden Vernetzungen innerhalb der deutschsprachigen Psychologie stellen aus unserer Sicht einen entscheidenden Erfolgsfaktor für unser Fach dar. Es gibt jedoch auch erhebliche Unterschiede hinsichtlich Studium und Berufssituation, die in den folgenden Beiträgen zu der Situation der Psychologie in Österreich herausgearbeitet werden. Dieser Blick über die Landesgrenzen hinaus schafft daher neue Perspektiven und liefert auch teils überraschende Kontraste.

Der Vergleich zwischen Deutschland und Österreich zeigt, dass einige Dinge ähnlich sind - zum Beispiel die guten Berufschancen der Psychologie-Absolventen und -Absolventinnen. Auch die Situation des wissenschaftlichen Nachwuchses in Österreich ähnelt der in Deutschland, mit einer hohen Zahl subjektiv berichteter Überstunden, einer geringen Zahl unbefristeter Beschäftigungsverhältnisse und einer nur mittleren Arbeitszufriedenheit. Die teils schwierige Situation des wissenschaftlichen Nach- wuchses in Deutschland wurde 2017 in einem Diskussionsforum in der Psychologischen Rundschau (Rentzsch, Harzer \& Wolter, 2017) thematisiert und kürzlich in einem internen Diskussionsforum zur Bezahlung von Doktorandinnen und Doktoranden auf der DGPs-Webseite vertieft. Wir begrüßen es, dass dieses Thema auch in Österreich genauer betrachtet wird.

Viele Dinge unterscheiden sich jedoch auch deutlich zwischen Deutschland und Österreich. So gibt es in Österreich eine künstliche Trennung zwischen Klinischer Psychologie und Psychotherapie, die die Berufsausübung im ambulanten Bereich stark erschwert. In Österreich hat sich das Berufsfeld Psychotherapie nicht aus der akademischen Psychologie heraus entwickelt. Aufgrund zweier unabhängiger Gesetze - des Psychologengesetzes und des Psychotherapiegesetzes - wurden zwei unabhängige Berufe geschaffen, während gerade das neue Psychotherapie-Ausbildungsgesetz in Deutschland noch einmal explizit anerkennt, dass die Psychologie die Mutterwissenschaft der Psychotherapie ist. Andererseits zeigt der Ländervergleich aber zum Beispiel auch, dass die Gesundheitspsychologie im österreichischen Gesundheitssystem eine wesentlich wichtigere Rolle einnimmt, als dies in Deutschland momentan der Fall ist.

Die Situation in Studium und Lehre ist in Österreich stark dadurch geprägt, dass viele junge Menschen aus Deutschland aufgrund des strikten NCs in unserem Fach ins Nachbarland ausweichen und dass die österreichischen Universitäten in Relation zu ihrer personellen Ausstattung eine deutlich höhere Anzahl an Studierenden aufnehmen müssen als deutsche Universitäten. Dies bringt 
Österreich in eine besondere Situation, sowohl was die Aufrechterhaltung von Qualitätsansprüchen an das Studium betrifft, als auch im Hinblick auf die Versorgung mit psychologischem Nachwuchs für verschiedene Berufsfelder inklusive der Wissenschaft. Da die Mehrzahl der Studienabschlüsse im Fach Psychologie in Österreich von Deutschen erworben wird und von diesen wiederum die Mehrzahl nach Deutschland zurückkehrt, ist eine Quotenregelung - wie neuerdings für die Medizin eingeführt immer wieder im Gespräch. Inwiefern diese Situation durch die Veränderungen der Psychologie-Studiengänge in Deutschland als Folge des neuen PsychotherapieAusbildungsgesetzes beeinflusst wird, gilt es abzuwarten. Wenn es in Zukunft nicht mehr möglich sein wird, mit einem österreichischen Studium die Voraussetzungen für die psychotherapeutische Approbation in Deutschland zu erwerben, ist von deutlichen Veränderungen in der Nachfrage von Studienplätzen durch deutsche Studierende auszugehen. Hieraus können sich Möglichkeiten für gezielte Veränderungen im Psychologiestudium in Österreich ergeben; es ist daher auch aus diesem Grunde ein günstiger Zeitpunkt für eine systematische Betrachtung der aktuellen Studiensituation, wie sie in diesem Themenheft vorgenommen wird.

Die Geschicke der österreichischen und deutschen Psychologie bleiben eng miteinander verwoben. Die Antwort auf die Frage "Tu felix Austria?" wird somit für unsere österreichischen Kolleginnen und Kollegen nicht zuletzt auch davon abhängen, wie Österreich auf die Veränderungen der deutschen Psychologiestudiengänge reagieren wird. Der Vorstand der DGPs dankt den Autorinnen und Autoren des Themenhefts, dass sie diese wichtigen Aspekte thematisieren und auf die aktuelle Agenda bringen. Wir hoffen, dass die gute und enge Zusammenarbeit auch in Zukunft so weitergeführt wird und würden uns freuen, wenn Wien in naher Zukunft die Kongresse von DGPs und ÖGP zusammenführen würde!

\section{Literatur}

\footnotetext{
Rentzsch, K., Harzer, C. \& Wolter, I. (2017). Stellungnahme zur Lage des wissenschaftlichen Nachwuchses in der Psychologie. Psychologische Rundschau, 68, 251-263.
}

Prof. Dr. Christian Fiebach

Institut für Psychologie

Goethe-Universität Frankfurt am Main

Theodor W. Adorno-Platz 6

60323 Frankfurt am Main

fiebach@psych.uni-frankfurt.de 